\title{
Characterizing cytomegalovirus infection one cell at a time
}

\author{
Systematic single-cell analyses of human cytomegalovirus infection reveal that host factors modulate the \\ progression of infection, but the course of viral infection itself is determined by virus genes.
}

\section{Laurent Perez}

1 uman cytomegalovirus (HCMV) infection can be life threatening, and vertical virus transmission during pregnancy is one of the leading cause of congenital birth defects ${ }^{1,2}$. However, treatment and prevention options are limited. HCMV has the largest known genome of human herpesviruses, with more than $235 \mathrm{kbp}$ of double-stranded DNA (dsDNA). In addition, HCMV has alternative transcript start sites, enabling strict temporal control of gene expression and the possibility of generating multiple distinct proteins from a single genomic locus. Host cellular heterogeneity and differences in infection kinetics further add to the complexity, and thus most previous screens of virus-host interactions studied the overall response to knocking out HCMV or host factors during infection ${ }^{3}$. To decipher HCMV-host cell interactions more thoroughly, a study ${ }^{4}$ published in Nature Biotechnology combines single-cell sequencing and CRISPR-based genetic perturbation to determine the contribution of viral and host factors during the HCMV lytic cycle in human primary cells. Whilst the methodology has previously been used to study oncogenesis, this is the first time that it has been applied to characterise virus-host interactions.

First, to clarify the organisation of the HCMV genome, the authors introduced a guide-RNA library targeting viral genes into primary human foreskin fibroblasts engineered to express Cas9, which generates cuts at defined positions along the viral genome following infection. In population-level analyses, these experiments revealed that the viral genes are arranged as functional modules. The terminal repeat regions contained no genes essential for viral replication, and targeting those regions had only a limited impact. By contrast, targeting the UL48A-UL73 and UL96-UL150 regions strongly affected viral function and protected the host cells, which is expected, as these regions

a Peturb-seq guide library

b Transcript with CRISPR-mediated perturbation

c Single-cell transcriptome

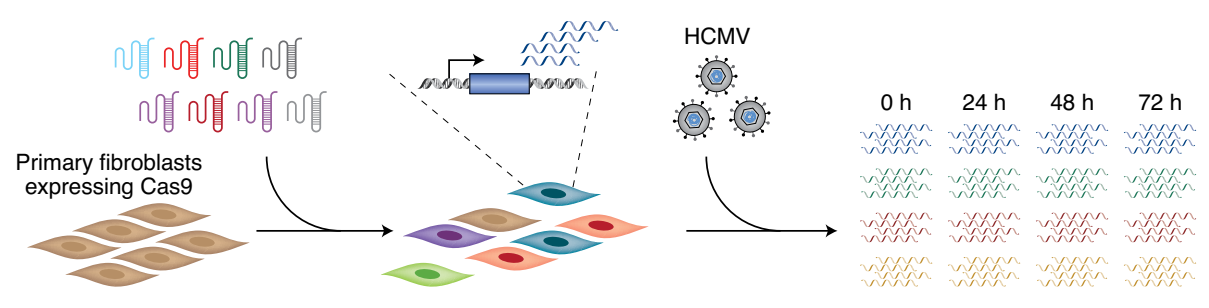

d Computational analysis

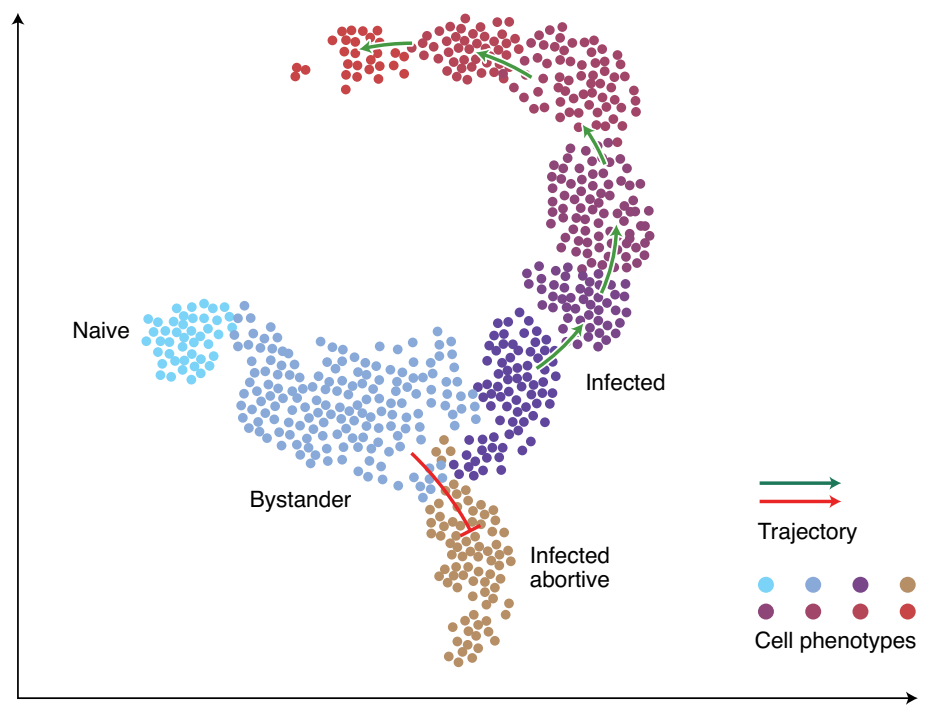

Fig. 1 | Perturbation single-cell sequencing analysis of HCMV infection. a, Human primary foreskin fibroblasts expressing the Cas9 enzyme were transduced with lentiviruses encoding selected guide RNAs targeting host and viral genes. b, Fibroblasts with transcript perturbation are infected with HCMV. c, Single-cell transcriptomic analysis is performed at different time points during the virus life cycle. $\mathbf{d}$, Computational analysis enables classification of cells according to their phenotype (naive, bystander and infected) and shows a trajectory of infection through different states or abortive infection.

contain factors necessary for replication, packaging and nucleocytoplasmic transport. Surprisingly, targeting the UL32-UL47 genes, which also encode factors essential for viral replication, results in an increased ability of the virus to kill the cells; while UL36, UL38 and UL40 are known to encode anti-apoptotic factors, this phenotype was also observed for other genes without known anti-apoptosis function. However, the in vivo relevance of this finding is unclear. Finally, targeting some of the genes encoding structural proteins did not result in phenotypic modifications. 
This unexpected finding means that the production of progeny virions and host cell death may be decoupled. Furthermore, the authors' approach enabled the identification of functional 'modules' in a viral genome for the first time.

In host-directed pooled genome-wide screens, the authors identified host factors involved in HCMV infection that had not previously been reported, including proteins involved in vesicle trafficking such as RAB6A, RIC1/KIAA1432, RGP1 and members of the conserved oligomeric Golgi (COG) complex, which function in internalization pathways. Knockdown or knockout of genes associated with lysosome positioning, such as the LAMTOR/Ragulator complex ${ }^{5}$, were also protective. Their screen also validated known factors that affect HCMV entry in fibroblasts, such as the PDGFR $\alpha$ receptor, members of the Cullin/RING pathway ${ }^{6}$ necessary for host protein degradation and genes involved in ER-associated degradation and in heparan sulfate biosynthesis ${ }^{7}$. Host factors that increase cell death were also identified, including genes involved in innate antiviral responses induced by interferon (IFN) and members of the PML nuclear bodies family known to promote the rapid condensation of herpes viral DNA into transcriptionally inactive heterochromatin.

A limitation of pooled genome-scale screens is that they lack temporal and cellular resolution. Single-cell transcriptomics of HCMV-infected cells revealed an inverse correlation between type I IFN and viral gene expression, which led the authors to define three main cellular populations: naive cells, bystander cells uninfected but IFN-positive and infected cells expressing different levels of viral genes but no IFN. Of note, these populations followed the expected cell cycle profile: cells were mainly in G1 phase for early infection, while during late infection, cells had an $S$ phase profile ${ }^{8}$. At the start of the experiment, most cells were in the naive population, and 6 hours later, most of the cells were either in the bystander or infected clusters. The infected cluster contained a trajectory of cellular states, which included six discrete steps. Surprisingly, only a minority of cells $(<1 \%)$ did not follow this common trajectory and experienced abortive infection due to high IFN- $\beta$ levels 9 .

To further define the interplay between host and viral factors, the authors used Perturb-seq analysis, which is a single-cell transcriptome and whole-genome CRISPR-based screen (Fig. 1). Perturb-seq enables the identification of individual gene targets, gene signatures and cell states affected by individual perturbations, as well as their genetic interactions ${ }^{10}$. Surprisingly, genetic perturbation of crucial host factors identified in the pooled screens could halt, delay or accelerate progression along the infection trajectory but did not fundamentally change the cell's trajectory. By contrast, targeting viral factors through Perturb-seq strongly influenced infection, with three different possible outcomes. Perturbation of genes in the UL35-UL43 region enhanced viral gene expression and reduced expression of some long noncoding RNAs, leading to host cell apoptosis.

Targeting of the UL52-ORFL152C region, encoding factors essential for viral genome replication, resulted in an abortive infection with low viral titres. Perturbation of the UL115-UL148 region, involved in the late part of the viral cycle, generated a mildly or unperturbed trajectory of infection similar to that following host factor perturbation. These findings led the authors to conclude that host genes mainly modulate progression through a deterministic infection program encoded by the viral genome. Whether this finding is broadly relevant to other viral infections remains to be seen. Irrespective of generalizability, this application of Perturb-seq highlights the crucial role of viral genes and identifies targets for antiviral and vaccine development, although hits from the screen will have to be confirmed and evaluated mechanistically.

The broad tropism of HCMV clinical strains enables the infection of many different cell types besides fibroblasts, and in some of those cell types, HCMV infection does not depend on the same receptors or internalization pathways ${ }^{7}$. Future work will be needed to understand the similarities and differences between HCMV entry, lytic cycle and latency in different cell types, such as human umbilical vein endothelial cells and $\mathrm{CD} 14^{+}$monocytes.

Perturb-seq methods will enable a better understanding of host-pathogen interactions at the single-cell level for diverse viral, bacterial and parasitic pathogens.

\section{Laurent Perez (iD)}

Section of Allergy and Immunology, Department of Medicine, Center for Human Immunology Lausanne (CHIL), University of Lausanne (UNIL) and Lausanne University Hospital (CHUV), Epalinges, Switzerland.

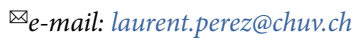

Published online: 25 October 2021

https://doi.org/10.1038/s41564-021-00994-w

References

1. Griffiths, P. \& Reeves, M. Nat. Rev. Microbiol. https://doi. org/10.1038/s41579-021-00582-z (2021).

2. Schleiss, M. R. Contemp. Pediatr. 35, 16-24 (2018).

3. Stern-Ginossar, N. et al. Science 338, 1088-1093 (2012).

4. Hein, M. Y. \& Weissman, J. S. Nat. Biotechnol. https://doi. org/10.1038/s41587-021-01059-3 (2021).

5. Filipek, P. A. et al. J. Cell Biol. 216, 4199-4215 (2017).

6. Harper, J. W. \& Schulman, B. A. Annu. Rev. Biochem. 90, 403-429 (2021).

7. Nguyen, C. C. \& Kamil, J. P. Viruses https://doi.org/10.3390/ v10120704 (2018)

8. Salvant, B. S., Fortunato, E. A. \& Spector, D. H. J. Virol. 72, 3729-3741 (1998).

9. Taylor, R. T. \& Bresnahan, W. A. J. Virol. 79, 3873-3877 (2005)

10. Dixit, A. et al. Cell 167, 1853-1866.e17 (2016).

Competing interests

The author declares no competing interests. 